\title{
Extraction of Singlet States from Noninteracting High-Dimensional Spins
}

\author{
F. Ciccarello, ${ }^{1,2,3}$ M. Paternostro, ${ }^{3}$ M. S. Kim, ${ }^{3}$ and G. M. Palma ${ }^{2}$ \\ ${ }^{1}$ CNISM and Dipartimento di Fisica e Tecnologie Relative, Università degli Studi di Palermo, Viale delle Scienze, Edificio 18, \\ I-90128 Palermo, Italy \\ ${ }^{2}$ NEST-INFM (CNR) and Dipartimento di Scienze Fisiche ed Astronomiche, Università degli Studi di Palermo, Via Archirafi 36, \\ I-90123 Palermo, Italy \\ ${ }^{3}$ School of Mathematics and Physics, Queen's University, Belfast BT7 1NN, United Kingdom
}

(Received 24 October 2007; published 18 April 2008)

\begin{abstract}
We present a scheme for the extraction of singlet states of two remote particles of arbitrary quantum spin number. The goal is achieved through post-selection of the state of interaction mediators sent in succession. A small number of iterations is sufficient to make the scheme effective. We propose two suitable experimental setups where the protocol can be implemented.
\end{abstract}

PACS numbers: 03.67.Mn, 03.67.Hk, 42.50.Pq, 73.23.-b

Achieving control at the quantum level is a pivotal requirement for the grounding of quantum technology and the development of reliable protocols for information processing. Frequently, state manipulation of a quantum device needs the connection of remote nodes of a network and the creation of their entangled state. Such a delocalized architecture has received strong experimental attention, especially at the quantum optics level. The heralded entanglement of remote atomic ensembles or individuallytrapped ions has been produced, and atom-photon entanglement has been observed [1]. The transfer of prebuilt entanglement to distant systems has been proposed as a way to distribute quantum channels [2].

A different approach exploits a mediated interaction between two remote nodes, 1 and 2, by means of their sequential coupling to the same ancillary system $e$ : The ancilla can bring to system 2 the information that has been previously impressed on it by its interaction with system 1 . Recently, this idea has been used in a solid-state context involving multiple electron scattering between magnetic impurities [3-5]. Interestingly, $e$ can also be used so as to condition the state of 1 and 2 . Once a three-body correlated state is established by means of bilocal $1-e$ and $2-e$ interactions, by measuring the state of $e$ we could project the remote systems onto entangled states with a nonzero probability [3-6]. In these examples, 1 and 2 are embodied by two-level systems whose finite Hilbert space bounds the entanglement that can be shared [7]. Overcoming such a limitation is an important task deserving attention.

Here we present a scheme that allows the "extraction" of maximally entangled states via an effective nondemolition Bell measurement performed onto the state of two spin- $s$ particles. This occurs through repeated injection and post-selection of simple mediators, each undergoing multiple scattering and spin-flipping between the two spins [8]. Besides achieving the maximum number of ebits allowed to two spin- $s$ systems, the protocol provides a procedure for accumulating entanglement. Remarkably, our protocol does not require interaction-time tuning. In our scheme maximal entanglement is stable against the parameters of the conditioned dynamics, which is a clear advantage in experimental implementations. In order to fix the ideas, we first describe the protocol in terms of a system composed of a conduction electron and two magnetic impurities. This will allow us to clearly illustrate the relevant features of our scheme. Later, we show how a cavity-quantum electrodynamics (QED) system, consisting of two multilevel atoms interacting with a photon field, can also embody the desired dynamics and allows a prompt experimental implementation.

We consider a quasi one-dimensional (1D) wire, such as a semiconductor quantum wire [9] or a single-wall carbon nanotube [10], where two identical spin-s magnetic impurities 1 and 2 are embedded at positions $x_{1}=0$ and $x_{2}=$ $x_{0}$ [see Fig. 1(a)]. Left-incident single electrons undergo multiple scattering between the two impurities and simultaneous spin-flipping. Assuming that the electron's coherence length exceeds $x_{0}$ and that each electron occupies only the lowest sub-band, the Hamiltonian reads (we set $\hbar=1) \quad \hat{H}=\hat{p}^{2} /\left(2 m^{*}\right)+J \hat{\boldsymbol{\sigma}} \cdot\left[\hat{\mathbf{S}}_{1} \delta(x)+\hat{\mathbf{S}}_{2} \delta\left(x-x_{0}\right)\right]$. Here, $\hat{p}=-i \nabla, m^{*}$, and $\hat{\boldsymbol{\sigma}}$ are the electron momentum, effective mass, and Pauli spin operator, respectively. $\hat{\mathbf{S}}_{i}$ is the spin- $s$ operator of the impurity $i=1,2$, and $J$ is the Heisenberg exchange coupling constant whose dimensions are frequency times length. Because of the elastic nature of the interactions, the energy spectrum reads $E=k^{2} / 2 m^{*}$

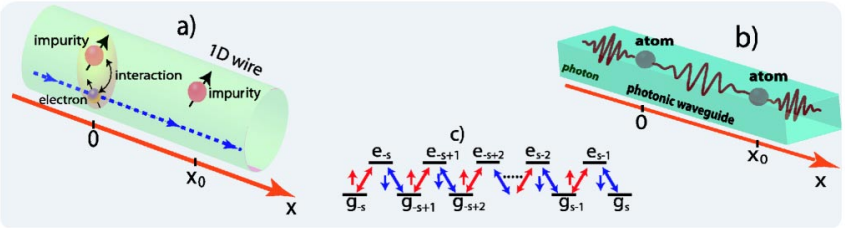

FIG. 1 (color online). Setups for the implementation of our scheme in nanowire (a) and cavity QED (b). (c) Multilevel atom embodying a spin-s particle for the setup in panel (b), where symbols $\uparrow, \downarrow$ indicate, abstractly, proper polarization of a photon. 
( $k$ is the electron wave vector). We label with $\hat{\mathbf{S}}=\hat{\boldsymbol{\sigma}}+$ $\hat{\mathbf{S}}_{1}+\hat{\mathbf{S}}_{2}$ the total spin of the system, while $m_{i}$ and $m_{e}=$ $\pm 1 / 2$ are the quantum numbers associated with $\hat{S}_{i z}$ and $\hat{\sigma}_{z}$, respectively. From now on, we denote $\{1 / 2,-1 / 2\}$ by $\{\uparrow, \downarrow\}$ and, for convenience, we use the basis of product states $\left|m_{e},\left\{m_{i}\right\}\right\rangle=\left|m_{e}\right\rangle_{e}\left|m_{1}, m_{2}\right\rangle_{12}$. We prepare the impurities in $\left|\left\{m_{i}^{\prime}\right\}\right\rangle_{12}$. An incoming electron of wave vector $k$ and spin state $\left|m_{e}^{\prime}\right\rangle_{e}$ is reflected (transmitted) in the state $\left|m_{e}\right\rangle_{e}$, while the impurities' spin state changes into $\left|\left\{m_{i}\right\}\right\rangle_{12}$ with probability amplitude $r(t)$ (we omit the dependence of $r$ and $t$ on $m_{e(i)}$ and $\left.m_{e(i)}^{\prime}\right)$. As $\hat{S}_{z}$ is a constant of motion, the only nonzero amplitudes are those obeying the selection rule $m_{12}^{\prime}+m_{e}^{\prime}=m_{12}+m_{e}$ with $m_{12}=m_{1}+m_{2}$. We solve this scattering problem by finding the steady states $\left|k, m_{e}^{\prime},\left\{m_{i}^{\prime}\right\}\right\rangle \quad$ with input part $\left\langle x \mid k, m_{e}^{\prime},\left\{m_{i}^{\prime}\right\}\right\rangle_{\text {in }}=$ $e^{i k x} \theta(-x)\left|m_{e}^{\prime},\left\{m_{i}^{\prime}\right\}\right\rangle$, where $\theta(x)$ is the Heaviside step function. Their output part reads $\left\langle x \mid k, m_{e}^{\prime},\left\{m_{i}^{\prime}\right\}\right\rangle_{\text {out }}=$ $\sum_{\alpha=r, t}\left\langle x \mid k, m_{e}^{\prime},\left\{m_{i}^{\prime}\right\}\right\rangle_{\alpha} \quad$ with $\quad\left\langle x \mid k, m_{e}^{\prime},\left\{m_{i}^{\prime}\right\}\right\rangle_{\alpha}=$ $\sum_{1} m_{e},\left\{m_{i}\right\} \alpha f_{\alpha}(x)\left|m_{e},\left\{m_{i}\right\}\right\rangle \quad$ and $\quad f_{\alpha}(x)=e^{i \eta_{\alpha} k x} \theta\left(\eta_{\alpha} x-\right.$ $\left.\frac{1+\eta_{\alpha}}{2} x_{0}\right)\left(\eta_{r}=-\eta_{t}=-1\right)$. The steady states are computed at all orders in $J$ solving the time-independent Schrödinger equation and imposing the matching of the wave function at $x_{i}$ 's [4]. We now derive how an (in general mixed) initial state of the impurities $\rho_{12}$ is transformed after scattering of an electron incoming in an arbitrary statistical mixture $\rho_{e}$ of the spin states $|\uparrow\rangle_{e}$ and $|\downarrow\rangle_{e}$. To this aim, we consider the state having $|k\rangle\langle k| \rho_{e} \rho_{12}$ as the input part, where $\langle x \mid k\rangle=e^{i k x} \theta(-x)$. The output part of such state is found by expanding it in the basis $\left\{\left|k, m_{e}^{\prime},\left\{m_{i}^{\prime}\right\}\right\rangle\right\}$ and replacing each component of this expansion with the corresponding output part. A further projection onto the electron's position eigenstates far from the impurities $\left|x_{r}\right\rangle$ and $\left|x_{t}\right\rangle\left(x_{r} \ll 0, x_{t} \gg x_{0}\right)$ yields $\sum_{\alpha=r, t}\left\langle x_{\alpha} \mid k, m_{e}^{\prime},\left\{m_{i}^{\prime}\right\}\right\rangle_{\alpha}\left\langle k, m_{e}^{\prime},\left\{m_{i}^{\prime}\right\} \mid x_{\alpha}\right\rangle\left|x_{\alpha}\right\rangle\left\langle x_{\alpha}\right|$. After tracing over the electron's degrees of freedom, the impurities' state becomes

$$
\mathcal{E}_{\rho_{e}}\left(\rho_{12}\right)=\sum_{\mu, \nu=\uparrow, \downarrow} \rho_{e \nu \nu}\left(\hat{R}_{\nu}^{\mu} \rho_{12} \hat{R}_{\nu}^{\mu \dagger}+\hat{T}_{\nu}^{\mu} \rho_{12} \hat{T}_{\nu}^{\mu \dagger}\right),
$$

where $\sum_{\mu}\left(\hat{R}_{\nu}^{\mu \dagger} \hat{R}_{\nu}^{\mu}+\hat{T}_{\nu}^{\mu \dagger} \hat{T}_{\nu}^{\mu}\right)=\mathbb{1}_{12}$. Each Kraus operator $R_{\nu}^{\mu}\left(T_{\nu}^{\mu}\right)$ depends only on $r$ 's ( $t^{\prime}$ 's) and is physically interpreted as the effect on $\rho_{12}$ due to the detection in spin-state $|\mu\rangle_{e}$ of a reflected (transmitted) electron incoming in state $|\nu\rangle_{e}$. We want to show that, conditioning the map in Eq. (1) and iterating it for $n$ electrons (injected in succession in the same spin state), singlet-state extraction is efficiently performed. To achieve this, we first describe what is induced by post-selecting the state of $n=1$ scattered electrons. Preparation and post-selection of a given electron-spin state, say $|\uparrow\rangle_{e}$, can be accomplished using spin-filtering contacts at the input or output ports of the wire [11], each selecting the same spin state. We obtain the final impurities' state $\varrho_{12}^{(1)}=\mathcal{E}_{\Uparrow \uparrow}\left(\rho_{12}\right)=\left(\hat{R}_{\uparrow}^{\dagger} \rho_{12} \hat{R}_{\uparrow}^{\dagger \dagger}+\hat{T}_{\uparrow}^{\dagger} \rho_{12} \hat{T}_{\uparrow}^{\dagger \dagger}\right) /$ $P_{\Uparrow}^{(1)}\left(\rho_{12}\right) \quad$ with success probability $P_{\Uparrow}^{(1)}\left(\rho_{12}\right)=$ $\operatorname{Tr}_{12}\left(\hat{R}_{\uparrow}^{\dagger} \rho_{12} \hat{R}_{\uparrow}^{\dagger \dagger}+\hat{T}_{\uparrow}^{\dagger} \rho_{12} \hat{T}_{\uparrow}^{\dagger \dagger}\right)$. The state $\varrho_{12}^{(n)}$ corresponding to $n$ electrons being prepared and post-selected in $|\uparrow\rangle_{e}$ is obtained as $\varrho_{12}^{(n)}=\mathcal{E}_{\Uparrow}^{n}\left(\rho_{12}\right)$ with conditional probability $P_{\Uparrow}^{(n \geq 1)}\left(\rho_{12}\right)=\prod_{j=1, n} P_{\Uparrow}\left(\varrho_{12}^{(j-1)}\right) \quad$ and $\quad \varrho_{12}^{(0)}=\rho_{12} \quad[12]$. Here, the rate of electron-injection is chosen so that, as an electron reaches the impurities, the previous one has been already scattered off.

Let $\left|\Psi_{s}^{-}\right\rangle$be the singlet state of two spin-s impurities. Using resonance conditions (i.e., $k x_{0} / \pi \in \mathbb{Z}$ ), in Figs. 2(a) and 2(b) we consider the case $s=1 / 2$ and plot the fidelity $F^{(n)}$ of $\varrho_{12}^{(n)}$ with respect to the singlet $\left|\Psi_{1 / 2}^{-}\right\rangle$together with $P_{\Uparrow}^{(n)}$ as functions of $n$ and $J / v$ for the initial product state $|1 / 2,-1 / 2\rangle_{12}\left(v=k / m^{*}\right.$ is the electronic group velocity). Clearly, $F^{(n)} \rightarrow 1$ for a range of values around $J / v \simeq 1.5$ that becomes a plateau when $n$ increases $(n<7$ iterations are enough to get fidelity higher than 0.95). For a fixed value of $J / v$, such convergence is exponential in $n$. Remarkably, although our protocol is conditioned on the outcomes of $n$ projective measurements all with the same outcome, the probability of success converges exponentially to 0.5 . Differently from [3-5], the scheme is still efficient for a nonoptimal $J / v$. Only a larger $n$ is required, for a fixed $s$. Moreover, the process is robust against discrepancies of $k$ with respect to resonance conditions and the use of a stream of mediators with mutually different wave vectors. In fact, by considering a Gaussian distribution of wave vectors centered at $k$ with variance $\sigma$, we have found that the fidelity (probability) is larger than 0.9 (0.35) for $k x_{0} \in[0.9,1.03] \pi$ and $\sigma / k$ up to $\simeq 5 \%$.

We now address the dependence of our figures of merit on the dimensionality of the impurities' spin. While the optimum ratio $J / v$ depends slightly on $s$, the efficiency of singlet extraction persists, as shown in Fig. 2(c) for
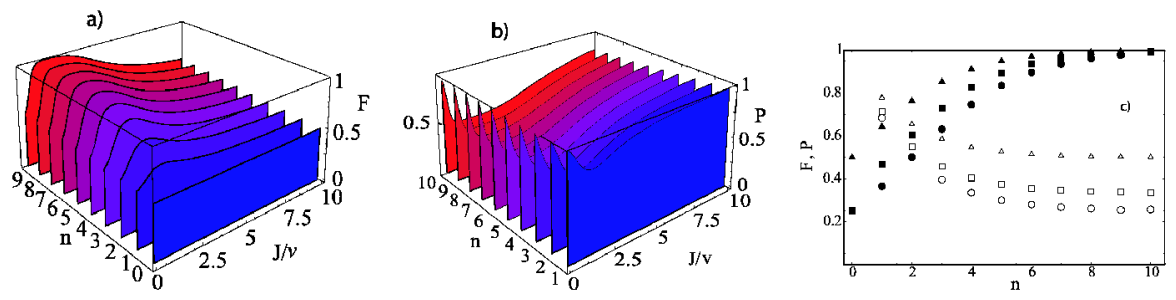

FIG. 2 (color online). (a) and (b) Fidelity and success probability vs. $J / v$ and $n$ for $s=1 / 2$. (c) $F^{(n)}$ (filled symbols) and $P_{\Uparrow \Uparrow}^{(n)}$ (empty symbols) vs $n$ for $s=1 / 2$ and $J / v=1.5(\boldsymbol{\Delta}, \triangle), s=1$ and $J / v=1.2(\boldsymbol{\square}, \square)$, and $s=3 / 2$ and $J / v=1.1(\bigcirc, \mathbf{O})$ at $k x_{0} / \pi \in \mathbb{Z}$ $(J / v$ is optimized for each $s)$. 
$\rho_{12}=|s,-s\rangle\langle s,-s|$ with $s=1 / 2,1,3 / 2$. Evidently, $\varrho_{12}^{(n)}$ rapidly converges to the singlet state regardless of $s$ (for instance, $F^{(n>5)}>0.95$ for $\left.s=1\right)$ while $P_{\Uparrow}^{(n)}$ approaches a finite value according to $P_{\Uparrow}^{(n \gg 1)}\left(\rho_{12}\right) \rightarrow\left|\left\langle\Psi_{s}^{-} \mid s,-s\right\rangle\right|^{2}=$ $(2 s+1)^{-1}$, exponentially in $n$. Our scheme thus asymptotically performs an effective projective measurement onto the spin- $s$ singlet state. As the singlet state has the maximum number of ebits allowed by the dimension of the Hilbert space of each impurity, the scheme provides a way to extract more than one ebit by considering sufficiently high-dimensional impurities' spins. Moreover, an entanglement accumulation mechanism is achieved [7]. For instance, for $s=2$ and $J / v=1$ the impurities' entanglement [measured by the logarithmic negativity, which is upper-bounded by $\log _{2}(d)$ for a $d^{2}$-dimensional Hilbert space] after $n=2,4$, and 5 is, respectively, 1.2, 1.8, and 2 . These are larger than the bound given by $\log _{2}(2 s+1)$ for $s=1 / 2,1$, and $3 / 2$, making our system an iteratively exploitable quantum channel: The impurities' entanglement can be extracted to many pairs of qubits [7]. Similar results hold for any initial eigenstate of $\hat{S}_{12 z}=$ $\hat{S}_{1 z}+\hat{S}_{2 z}$ with null eigenvalue.

We now show how the efficiency of singlet-state extraction relies on resonance-induced selection rules. Let $\left|s, s, s_{12}, m_{12}\right\rangle$ be the coupled basis of common eigenstates of $\hat{\mathbf{S}}_{1}^{2}, \hat{\mathbf{S}}_{2}^{2}, \hat{\mathbf{S}}_{12}^{2}$, and $\hat{S}_{12 z}$ (the singlet state thus reads $\left|\Psi_{s}^{-}\right\rangle=$ $\left.\left|s, s, s_{12}=0, m_{12}=0\right\rangle\right)$. Let $\mathcal{E}_{\uparrow}\left(\rho_{12}\right)$ be the unconditioned map in Eq. (1) for $\rho_{e}=|\uparrow\rangle_{e}\langle\uparrow|$. Clearly, with the additional output-filtering of $|\uparrow\rangle_{e}, \mathcal{E}_{\uparrow}\left(\rho_{12}\right)$ becomes $\mathcal{E}_{\uparrow}\left(\rho_{12}\right)$. Notice that in general the product state $|s, s\rangle_{12}$ is the only fixed point of $\mathcal{E}_{\uparrow}\left(\rho_{12}\right)$. However, at resonance $\left(k x_{0}=n \pi\right), \hat{\mathbf{S}}_{12}^{2}$ is conserved due to the equal probabilities of the electron to be found at each of the $x_{i}$ 's [4]. Thus, repeated applications of the unconditioned map cannot drive the system out of the eigenspace associated with a set value of $s_{12}$. This and the conservation of $\hat{S}_{z}$ imply that the singlet-state $\left|\Psi_{s}^{-}\right\rangle$ becomes an additional fixed point of $\mathcal{E}_{\uparrow}$. Let $p_{s_{12}}$ be the probability for an injected electron prepared in $|\uparrow\rangle_{e}$ to be flipped down when the impurities are prepared in $\left|s, s, s_{12}, 0\right\rangle$. The selection rules at resonance yield the evolved impurities' state $p_{s_{12}}\left|s, s, s_{12}, 1\right\rangle\left\langle s, s, s_{12}, 1\right|+$ $\left(1-p_{s_{12}}\right)\left|s, s, s_{12}, 0\right\rangle\left\langle s, s, s_{12}, 0\right|$. If we post-select $|\uparrow\rangle_{e}$ at the output ports, each state $\left|s, s, s_{12}, 0\right\rangle$ with $s_{12} \neq 0$ is left unchanged with probability $1-p_{s_{12}}$. Under application of $\mathcal{E}_{\Uparrow \Uparrow}^{n \gg 1}$, it thus vanishes as $\left(1-p_{s_{12}}\right)^{n \gg 1} \simeq 0$, which clarifies the exponential convergence exhibited by $F^{(n)}$ and $P_{\Uparrow}^{n}$ (cf. Fig. 2). Differently, $\left|s, s, s_{12}=0,0\right\rangle=\left|\Psi_{s}^{-}\right\rangle$survives to the application of $\mathcal{E}_{\Uparrow}^{n \gg 1}$ since the selection rules ensure that $p_{s_{12}=0}=0$ [4]. If we consider an element of the uncoupled basis $|\xi\rangle$ such that $\hat{S}_{12 z}|\xi\rangle_{12}=0$ and expand it over $\left|s, s, s_{12}, 0\right\rangle$ 's, we find that, under application of $\mathcal{E}_{\Uparrow}^{n \gg 1},|\xi\rangle\left\langle\xi|\rightarrow| \Psi_{s}^{-}\right\rangle$with a probability $P_{\Uparrow}^{(n \gg 1)}$ that asymptotically becomes $\left|\left\langle\Psi_{s}^{-} \mid \xi\right\rangle\right|^{2}$. When $|\xi\rangle=$ $|s,-s\rangle_{12}$, as in Fig. 2, the asymptotic probability is $(2 s+$
$1)^{-1}$. Our clear interpretation of the physics behind our protocol is an important feature for the development of novel schemes.

Unlike previous proposals [3-5], a remarkable advantage of our protocol is that it can be applied to magnetic impurities of spin higher than $1 / 2$. For instance, we could use a 1D semiconducting wire with embedded Mn impurities having $s=5 / 2$. Although impressive progress has been made, a major obstacle in spintronics implementations is the current lack of high-efficiency electron-spin filters [11]. As a way to overcome such difficulties, we discuss an alternative system [see Fig. 1(b)] able to act as an accurate simulator of $\hat{H}$ and holding the promises for not far-fetched experimental implementation. The basic idea is to replace the electron with a single photon propagating in a 1D photonic waveguide sustaining two frequency-degenerate orthogonally polarized modes. For consistency of notation, we denote circular polarizations by $\uparrow$ and $\downarrow$. Each impurity is now embodied by a multilevel atom [see Fig. 1(c)] having a $(2 s+1)$-fold degenerate ground level spanned by $\left\{\left|g_{-s}\right\rangle, \ldots,\left|g_{s}\right\rangle\right\}$ and a $2 s$-fold degenerate excited level spanned by $\left\{\left|e_{-s}\right\rangle, \ldots,\left|e_{s-1}\right\rangle\right\}$. The standard three-level $\Lambda$ and five-level $M$ configurations are recovered, for instance, by taking $s=1 / 2$ and $s=1$, respectively. Such a configuration may be found in the rich hyperfine spectrum of alkali atoms. We assume electricdipole selection rules such that each $\left|e_{m}\right\rangle$ ( $m=$ $-s, \ldots, s-1)$ is connected to the pair of nearest-neighbor ground states $\left\{\left|g_{m}\right\rangle,\left|g_{m+1}\right\rangle\right\}$ via coherent scattering of a photon between the two orthogonally polarized modes. To fix the ideas, we take the transition $\left|e_{m}\right\rangle \leftrightarrow\left|g_{m}\right\rangle\left(\left|e_{m}\right\rangle \leftrightarrow\right.$ $\left.\left|g_{m+1}\right\rangle\right)$ to be driven by the $\uparrow$-polarized ( $\downarrow$-polarized) mode. Each atom can thus undergo a transition between two adjacent ground states $\left|g_{m}\right\rangle \leftrightarrow\left|g_{m+1}\right\rangle$ via a twophoton Raman process with associated coherent scattering of a photon between states $|\uparrow\rangle$ and $|\downarrow\rangle$. Assuming a linear dispersion law $E=v_{\mathrm{ph}} k$ with $v_{\mathrm{ph}}$ the group velocity of the photon and $E$ its energy, the free Hamiltonian of the field in the waveguide is [13] $\hat{H}_{\mathrm{ph}}=$ $-i \sum_{\beta=R, L} \sum_{\gamma=\uparrow, \downarrow} \int d x v_{\beta} \hat{c}_{\beta, \gamma}^{\dagger}(x) \partial_{x} \hat{c}_{\beta, \gamma}(x)$ with $v_{R}=$ $-v_{L}=v_{\text {ph }}$ and $\hat{c}_{R, \gamma}^{\dagger}(x)\left[\hat{c}_{L, \gamma}^{\dagger}(x)\right]$ the bosonic operator creating a right (left) propagating photon of polarization $\gamma$ at position $x$. Considering dipole transitions with Rabi frequencies and natural excited-state linewidth smaller than the corresponding detuning from the excited state, each state $\left|e_{m}\right\rangle$ is only virtually populated and the effective atom-photon coupling reads $\hat{V}=$ $\sum_{i=1,2} \int d x\left[\hat{c}_{\uparrow}^{\dagger}(x) \hat{c}_{\downarrow}(x) \hat{\mathcal{S}}_{i-}+\right.$ h.c. $] \delta\left(x-x_{i}\right)$ with $c_{\gamma}^{\dagger}(x)=$ $\sum_{\beta=R, L} c_{\beta, \gamma}^{\dagger}(x)$ and $\hat{\mathcal{S}}_{i+}=\hat{\mathcal{S}}_{i-}^{\dagger}=\sum_{m=-s}^{s-1} J_{s, m}\left|g_{m+1}\right\rangle_{i}\left\langle g_{m}\right|$. Here $J_{s, m}$ is the effective transition rate of the Raman process leading the $i$ th atom from $\left|g_{m}\right\rangle_{i}$ to $\left|g_{m+1}\right\rangle_{i}$, assuming identical atoms. We map the photonic polarization into an effective pseudospin $s$ as $\hat{\boldsymbol{\sigma}}=\int d x \hat{\boldsymbol{\sigma}}(x)$ with $\hat{\sigma}_{+}(x)=\hat{\sigma}_{-}^{\dagger}(x)=c_{\uparrow}^{\dagger}(x) c_{\downarrow}(x)$ and $\hat{\sigma}_{z}(x)=\left[\hat{c}_{\uparrow}^{\dagger}(x) \hat{c}_{\uparrow}(x)-\right.$ $\left.\hat{c}_{\downarrow}^{\dagger}(x) \hat{c}_{\downarrow}(x)\right] / 2$. Provided that $J_{s, m}=J \chi_{s, m} \quad$ with 
$\chi_{s, m}=[s(s+1)-m(m+1)]^{1 / 2}$, each $\hat{\mathcal{S}}_{i+}$ becomes the effective pseudospin $s$ operator $\hat{\mathcal{S}}_{i \pm}=J \hat{S}_{i \pm}$, where $\hat{S}_{i \pm}$ obeys the standard algebra of angular momentum. Under these conditions, this model can be regarded as the second quantization version of $\hat{H}$ with the exchange electronimpurity coupling replaced by an isotropic $X Y$ interaction. It is easily checked that $\left[\hat{H}_{\mathrm{ph}}+\hat{V}, \hat{S}_{z}\right]=0$ and, provided $k x_{0} / \pi \in \mathbb{Z},\left[\hat{H}_{\mathrm{ph}}+\hat{V}, \hat{\mathbf{S}}_{12}^{2}\right]=0$. Through standard procedures [13], we have derived the stationary states $\left|k, m_{\mathrm{ph}}^{\prime},\left\{m_{i}^{\prime}\right\}\right\rangle$ for a single photon with wave vector $k\left(m_{\mathrm{ph}}^{\prime}\right.$ is the quantum number of $\hat{\sigma}_{z}$ ). The input (output) part of $\left|k, m_{\mathrm{ph}}^{\prime},\left\{m_{i}^{\prime}\right\}\right\rangle$ is formally analogous to $\left|k, m_{e}^{\prime},\left\{m_{i}^{\prime}\right\}\right\rangle_{\mathrm{in}}$ $\left(\left|k, m_{e}^{\prime},\left\{m_{i}^{\prime}\right\}\right\rangle_{\text {out }}\right)$. Here, $\mathcal{E}_{\Uparrow \Uparrow}\left(\rho_{12}\right)$ is obtained analogously to what is done for the previous model with photonic polarization detection used for the post-selection. Plots analogous to those in Figs. 2 are reproduced with only negligible quantitative differences. Practically, $\mathcal{E}_{\uparrow}\left(\rho_{12}\right)$ is obtained using Geiger-like photodetectors at the input or output ports of the waveguide combined with polarizing beam splitters to realize $\mathcal{E}_{\uparrow \uparrow}\left(\rho_{12}\right)$. Each $J_{s, m}$ depends on the product of the Clebsch-Gordan coefficients associated with the far-detuned (one-photon) transitions involved in the process $\left|g_{m}\right\rangle \leftrightarrow\left|g_{m+1}\right\rangle$. The condition $J_{s, m}=J_{\chi_{s, m}}$ is clearly fulfilled for $s=1 / 2$, involving only $\chi_{1 / 2,-1 / 2}=$ 1 . For $s \geq 1$ the pattern of $J_{s, m}$ 's might in general deviate from the ideal one dictated by the $\chi_{s, m}$ 's. However, we have assessed $F^{(n)}$ and $P_{\Uparrow \Uparrow}^{n}$ finding that our scheme is strikingly robust against such deviations [14]. For instance, for $s=3 / 2$, the ideal pattern yields $J_{3 / 2,1 / 2} / J_{3 / 2,-3 / 2}=1$ and $J_{3 / 2,-1 / 2} / J_{3 / 2,-3 / 2}=2 / \sqrt{3}$. By taking $J_{3 / 2,-3 / 2} / v_{\mathrm{ph}}=$ $J_{3 / 2,1 / 2} / v_{\mathrm{ph}}=\sqrt{3}$ and $J_{3 / 2,-1 / 2} / v_{\mathrm{ph}}=4 \sqrt{3}$, which are far from ideal, we obtain $F^{(n>6)}=0.97$, and $P^{(n>6)}=0.26$. These values are basically identical to the values obtained with the ideal ratios. This alternative model turns out to be also robust against deviations of $k$ from the ideal resonance conditions [14]. Our protocol is thus resilient and flexible to the actual working conditions.

For a realization of the scheme in the case $s=1 / 2$, the impurities can be embodied by $\Lambda$ configurations encompassed in the (single-electron charged) trionic picture of semiconducting quantum dots (QDs), which have been the center of extensive studies [15]. Positioning QDs within a waveguide or a cavity is now achievable with high accuracy $(\sim 30 \mathrm{~nm})$. A back-of-the-envelope calculation shows that for a photonic wavelength of $780 \mathrm{~nm}$ in a GaAs structure (400 $\mathrm{nm}$ in a $\mathrm{GaN}$ nanowire), $x_{0} \sim$ $0.1 \mu \mathrm{m}(1 \mu \mathrm{m})$ is required for the resonance condition, which is achievable. Strong coupling between a single QD and a cavity field has been demonstrated [15] and current experimental efforts make the achievement of $J / v \sim 1$ realistic in large refractive-index structures, without the need of a waveguide's band gap. We consider GaInN (InAs) QDs in GaN (GaAs) nanowires as potential candidates for our scheme. Their typical quality factor is $\simeq 10^{3}$, implying a single-photon lifetime $\tau_{p} \sim 1$ ps at $400 \mathrm{~nm}$ wavelength. The refractive index of $\mathrm{GaN}$ is $\sim 2$, so that a photon travels $x_{0}=1 \mu \mathrm{m}$ in $\tau_{p} / 100$. Ongoing experimental progress makes the controlled growth and positioning of two QDs in $\mu \mathrm{m}$-long waveguides quite realistic.

We have proposed a scheme for the conditional extraction of singlet states of two remote spin $s$ 's based on projective measurements over interaction mediators. The protocol does not require the demanding recycling of the same mediator. It achieves $s+1 / 2$ ebits with finite probability, a small number of steps, weak requirements on the parameters entering the dynamics, and no interaction-time tuning. We have proposed a realistic setup where the mediators are embodied by photons and the spins to be entangled by artificial atoms.

We thank M. Weber, G. Fishman, F. Julien, J.-M. Lourtioz, Y. Omar, R. Passante, L. Rizzuto, and M. Tchernycheva. We acknowledge support from PRIN 2006 "Quantum noise in mesoscopic systems," The Leverhulme Trust, EPSRC, QIPIRC, and the British Council/MIUR British-Italian Partnership Program 2007-2008.

[1] D. N. Matsukevich, et al., Phys. Rev. Lett. 96, 030405 (2006); B. Julsgaard et al., Nature (London) 432, 482 (2004); J. Volz et al., Phys. Rev. Lett. 96, 030404 (2006).

[2] M. Paternostro, W. Son, and M.S. Kim, Phys. Rev. Lett. 92, 197901 (2004).

[3] D. Yang, S.-J. Gu, and H. Li, arXiv:quant-ph/0503131; A. T. Costa, Jr., S. Bose, and Y. Omar, Phys. Rev. Lett. 96, 230501 (2006); G. L. Giorgi and F. De Pasquale, Phys. Rev. B 74, 153308 (2006).

[4] F. Ciccarello et al., New J. Phys. 8, 214 (2006); J. Phys. A 40, 7993 (2007); Laser Phys. 17, 889 (2007); F. Ciccarello, G. M. Palma, and M. Zarcone, Phys. Rev. B 75, 205415 (2007)

[5] K. Yuasa and H. Nakazato, J. Phys. A 40, 297 (2007).

[6] H. Nakazato, M. Unoki, and K. Yuasa, Phys. Rev. A 70, 012303 (2004); L.-A. Wu, D. A. Lidar, and S. Schneider, ibid. 70, 032322 (2004); G. Compagno et al., ibid. 70, 052316 (2004).

[7] M. Paternostro, M. S. Kim, and G. M. Palma, Phys. Rev. Lett. 98, 140504 (2007).

[8] For quantum state engineering via iterated quantum operations, see D. Burgarth and V. Giovannetti, New J. Phys. 9, 150 (2007).

[9] S. Datta, Electron Transport in Mesoscopic Systems (Cambridge University, Cambridge, England, 1997).

[10] S. J. Tans et al., Nature (London) 386, 474 (1997).

[11] D. D. Awschalom, D. Loss, and N. Samarth, Semiconductor Spintronics and Quantum Computation (Springer, Berlin, 2002).

[12] For the single-impurity case without spin-flip see W. Kim, R. K. Teshima, and F. Marsiglio, Europhys. Lett. 69, 595 (2005).

[13] J.-T. Shen and S. Fan, Phys. Rev. Lett. 95, 213001 (2005); 98, 153003 (2007).

[14] F. Ciccarello et al. (to be published).

[15] M. Atatüre et al., Science 312, 551 (2006); K. Hennessy, et al., Nature (London) 445, 896 (2007). 